\title{
A Subgroup of Latently Mycobacterium tuberculosis Infected Individuals Is Characterized by Consistently Elevated IgA Responses to Several Mycobacterial Antigens
}

\author{
Ralf Baumann, ${ }^{1,2}$ Susanne Kaempfer, ${ }^{2}$ Novel N. Chegou, ${ }^{1}$ Wulf Oehlmann, \\ Ralf Spallek, ${ }^{2}$ André G. Loxton, ${ }^{1}$ Paul D. van Helden, ${ }^{1}$ Gillian F. Black, ${ }^{1}$ \\ Mahavir Singh, ${ }^{2}$ and Gerhard Walzl ${ }^{1}$ \\ ${ }^{1}$ DST/NRF Centre of Excellence for Biomedical TB Research and MRC Centre for TB Research, Division of Molecular Biology and \\ Human Genetics, Department of Biomedical Sciences, Faculty of Medicine and Health Sciences, Stellenbosch University, P.O. Box \\ 19063, Tygerberg 7505, South Africa \\ ${ }^{2}$ Lionex Diagnostics and Therapeutics, Salzdahlumer Straße 196, 38126 Braunschweig, Germany
}

Correspondence should be addressed to Mahavir Singh; info@lionex.de

Received 21 May 2014; Revised 7 August 2014; Accepted 21 August 2014

Academic Editor: Kostas Spiropoulos

Copyright (C) 2015 Ralf Baumann et al. This is an open access article distributed under the Creative Commons Attribution License, which permits unrestricted use, distribution, and reproduction in any medium, provided the original work is properly cited.

\begin{abstract}
Elevated antibody responses to Mycobacterium tuberculosis antigens in individuals with latent infection (LTBI) have previously been linked to an increased risk for progression to active disease. Studies in the field focussed mainly on IgG antibodies. In the present study, IgA and/or IgG responses to the mycobacterial protein antigens AlaDH, NarL, $19 \mathrm{kDa}$, PstS3, and MPT83 were determined in a blinded fashion in sera from 53 LTBI controls, 14 healthy controls, and 42 active TB subjects. Among controls, we found that elevated IgA levels against all investigated antigens were not randomly distributed but concentrated on a subgroup of $<30 \%-$ with particular high levels in a small subgroup of 5\% comprising one progressor to active TB. Based on a specificity of $100 \%$, anti-NarL IgA antibodies achieved with $78.6 \%$ sensitivity the highest accuracy for the detection of active TB compared to healthy controls. In conclusion, the consistently elevated IgA levels in a subgroup of controls suggest higher mycobacterial load, a risk factor for progression to active TB, and together with high IgG levels may have prognostic potential and should be investigated in future large scale studies. The novel antigen NarL may also be promising for the antibody-based diagnosis of active TB cases.
\end{abstract}

\section{Introduction}

Approximately one third of the world's population has latent infection with Mycobacterium tuberculosis [1]. Latent M. tuberculosis infection (LTBI) represents a considerable reservoir of future active disease and contagion. Risk factors include co-infection with human immunodeficiency virus (HIV), diabetes mellitus, low body weight, old age, or use of immunosuppressive medications. In immunocompetent individuals, the annual risk of progression is estimated to be greatest in the first 1 or 2 years after infection.

Preventing LTBI individuals from reactivation (before they become symptomatic and infectious) may constitute a major step towards the elimination of TB. Therefore, the revised global plan to stop TB (2011-15) [2] has set 2015 as the goal for point-of-care tests that can be used for the accurate detection of preclinical TB.

Bacterial load is associated with disease risk, and antibody levels against $M$. tuberculosis components may be biomarkers for load [3] as well as disease risk [4-6]. Stratification of TB suspects into groups of absent, low (smear-negative tuberculosis), and high (smear-positive tuberculosis) bacterial burden showed that antibody levels correlated with bacillary burden [4]. In the same study, data of the macaque model, which reproduces key features of latent TB in humans, showed that infection outcome was reflected by the antibody response in the latent infection group [4], thereby confirming previous animal studies [7-9]. Although studies were small, 
increased specific antibody levels during the LTBI stage in humans also characterized progressors [5, 6]. Antibodybased tests for the diagnosis of active TB disease are often criticized for their lack of specificity in TB endemic regions [10], which is due to a high background prevalence of LTBI [11]. Clearly, further research is needed to elucidate whether M. tuberculosis specific antibody tests can determine active $\mathrm{TB}$ and cases at risk for progression and whether lack of specificity of antibody-based TB tests will turn out to be due to a high risk for an early stage of progression to active TB.

The low frequency of reactivation in immunocompetent LTBI individuals poses a challenge for the discovery of prognostic markers. Therefore, we chose to investigate the distribution of serologic responses, as a nonrandom distribution may point to a subgroup with higher bacterial load and an increased risk for future progression to disease. In a South African TB endemic population, we evaluated the serodiagnostic reactivity of L-alanine dehydrogenase $(\mathrm{AlaDH})(\mathrm{Rv} 2780)$, nitrate/nitrite response transcriptional regulator NarL (Rv0844c), periplasmic phosphate-binding lipoprotein PstS3 (Rv0928), $19 \mathrm{kDa}$ lipoprotein antigen precursor LpqH (Rv3763), and lipoprotein MPT83 (Rv2873). IgG responses to each of the two surface-exposed lipoproteins, $19 \mathrm{kDa}$ and MPT83, are predominantly recognized in active TB sera and not in non-TB disease (NTBD) sera [4]. The $19 \mathrm{kDa}$ antigen promotes binding to host cells and phagocytosis of mycobacteria [12], inhibits IFN- $\gamma$-induced killing of mycobacteria by macrophages [13], and induces macrophage apoptosis [14]. MPT83 elicits T cell proliferation of the majority of TB patients and is being considered as future subunit vaccine candidate [15]. The third lipoprotein, M. tuberculosis PstS3, which is involved in active transport of inorganic phosphate across the membrane (import), has not been investigated yet in subjects with LTBI for the serodiagnosis of M. tuberculosis [16]. However, PstS3 generates IFN$\gamma$-producing cells in a more potent manner than the closely related $38 \mathrm{kDa}$ (PstS1) [17], a major M. tuberculosis antigen [4, $18,19]$. The in TB serodiagnostics newly investigated protein antigen NarL is a putative nitrate response regulator involved in the regulation of anaerobic metabolism [20] and is part of the membrane fraction of $M$. tuberculosis [21]. IgG responses to the culture filtrate (and membrane) protein $\mathrm{AlaDH}$ are unable to distinguish untreated TB patients and controls in endemic settings [22]. AlaDH is present in M. tuberculosis but not in the vaccine strain Mycobacterium bovis BCG [21,23]. It may play a role in cell wall synthesis as L-alanine is an important constituent of the peptidoglycan layer.

We focused on IgA antibodies because M. tuberculosis-specific IgA antibodies discriminated better than IgG antibodies between active TB and TB endemic controls in Africa [24] as well as between healthy close contacts of pulmonary TB patients and healthy individuals without such contact [25]. Moreover, IgA production was reported to be highly T-cell dependent [26], and a protective role for IgA was suggested in several murine models of mycobacterial infection, for example, [27].

\section{Materials and Methods}

2.1. Study Population. Sera utilized to probe antibody assays were from a retrospective serum bank collected from individuals in an epidemiological field site in metropolitan Cape Town in South Africa with a population of whom $99.7 \%$ are of mixed race. The incidence of new smear-positive TB in this community was 341/100 000 population in 2002 and the majority of people harbours latent infection [28]. In the study community, BCG vaccination (Danish strain, 1331, Statens Serum Institute, Copenhagen, Denmark) is routinely administered at birth since 1971. The study was approved by the Ethics Committee of the Faculty of Health Sciences at the Stellenbosch University and written informed consent was obtained from all participants or their legal guardians in the case of children.

Community Control Subjects. Inclusion criteria for all healthy control participants enrolled into the study were residence in the described community, absence of clinical signs of TB or other diseases, absence of prior TB, HIV negativity, and no pregnancy. Several parameters were employed to determine TB infection status: the Mantoux skin test, two different and independent commercial interferon- $\gamma$ release assays (IGRAs) [the QuantiFERON TB Gold in Tube (QFT) (Qiagen, Hilden, Germany, Australia) and T-SPOT.TB (Oxford Immunotec, Abingdon, UK)], chest X-ray (CXR), and sputum AFB staining. We used $>15 \mathrm{~mm}$ as cut off for Mantoux test positivity [29] and alternatively $>5 \mathrm{~mm}$ [30] as described in more detail in the results section. Two IGRAs were used, as rates of positive results have been reported to differ between $\mathrm{T}$ SPOT.TB and QuantiFERON-TB Gold (e.g. [31, 32]). Sixtyfour consecutively recruited recent household contacts of active TB patients were part of a larger household contact study, and because the non-LTBI individuals constituted fewer than $20 \%$ of the contacts, three additional community controls with no known previous TB exposure were added and underwent the same investigations as the household contacts except the IGRA assays. The majority of LTBI subjects were followed up for the development of active TB disease within 2 years after their first recruitment, and one progressor (after 3 months) was identified according to hospital records.

TB Patients. Forty-two HIV-negative, Ziehl-Neelsen sputum smear-positive and BACTEC sputum culture-positive active pulmonary $\mathrm{TB}$ patients with no known multidrug resistance that were part of the same larger study as the controls, of which results have been published recently [33, 34], were included. Seven TB patients were excluded due to HIVseropositivity $(n=1)$, NTM infection $(n=2)$, or concomitant illness, such as diabetes mellitus $(n=4)$. All TB patients were self-reporting, untreated cases, and all except 2 had a first episode of active TB.

2.2. Serum Preparation. Blood samples of control subjects and TB patients (at diagnosis prior to initiation of treatment) were taken. After transport of the blood samples to the laboratory (within $2 \mathrm{~h}$ of collection and at ambient conditions), 
TABLE 1: Recombinant antigens of M. tuberculosis used in this study.

\begin{tabular}{lccccc}
\hline Protein name(s) & Rv number & Mol mass $(\mathrm{kDa})$ & Expression vector & 6-fold His-Tag & E. coli host strain \\
\hline NarL & Rv0844c & 23.9 & pET22 & N-terminal & BL21 (DE3) \\
AlaDH & Rv2780 & 38.7 & pJLA604 & - & CAG629 \\
19 kDa glycolipoprotein, LpqH & Rv3763 & 16.0 & pET26 & C-terminal & BL21 (DE3) \\
PstS3 & Rv0928 & 38.8 & pET22 & C-terminal & Rosetta (DE3) \\
MPT83, MPB83 & Rv2873 & 24.9 & pET21 & C-terminal & BL21 (DE3) [pLysS] \\
\hline
\end{tabular}

Nitrate/nitrite response transcriptional regulatory protein NarL; secreted L-alanine dehydrogenase (AlaDH); $19 \mathrm{kDa}$ lipoprotein antigen precursor LpqH; periplasmic phosphate-binding lipoprotein PstS3; cell surface lipoprotein MPT83.

serum was separated by centrifugation $(1250 \times \mathrm{g}$ for $7 \mathrm{~min})$ and stored in aliquots at $-80^{\circ} \mathrm{C}$ until use.

2.3. Antigen Cloning, Protein Expression, and Purification. The production of the functional M. tuberculosis Lalanine dehydrogenase $(\mathrm{AlaDH})$ in the heat-induced strain Escherichia (E.) coli CAG629 (pMSK12) has been described previously [35]. For cloning, the genes of the remaining 4 protein antigens (Table 1) were amplified by PCR using primers with integrated restriction sites allowing the sitedirected insertion of cleaved PCR-products into $\mathrm{pET}$ vectors (Novagen). All four genes were fused to sequence coding for 6-fold His-tag (Table 1). The genes were expressed in $E$. coli BL21(DE3) or in case of pstS3 in E. coli Rosetta (DE3). The antigens were purified using standard chromatographic methods (affinity chromatography, ion exchange chromatography, size exclusion chromatography). Insoluble antigens were solubilized (refolded) from denaturating conditions ( $8 \mathrm{M}$ urea) into buffers free of choatropic reagents. Further details regarding the protein purification are described in the supplements (see Supplementary Material available online at http://dx.doi.org/10.1155/2015/364758).

2.4. Enzyme-Linked Immunosorbent Assay. Microtiter plates were coated with $M$. tuberculosis antigens and serologic antibody responses were determined using standard procedures as described in the supplements. Laboratory personnel performing the serodiagnosis assays were blinded to the clinical status of the patients or the controls. Subsequent record reviews were done by clinical staff to classify the individuals into clinical groups without knowledge of the serologic response phenotype.

2.5. Statistical Analysis. GraphPad Prism (Graph Pad, San Diego, CA, USA) was used to create graphs and statistical analyses were performed using Medcalc software (Kagi, Berkeley, CA). The $t$-test for independent samples or the Mann-Whitney test was used for the statistical comparison of 2 groups depending on the fact whether or not the data were normally distributed. Given the aim to develop a diagnostic test for TB with a specificity level above at least $90 \%$, the experiments were analyzed by using specificity levels of $\geq 90 \%$, as was done by other investigators [36, 37]. The Spearman rank test was used for correlation analyses. Generally, a two-tailed $P$ value of $P \leq 0.05$ was considered significant.

\section{Results}

3.1. Classification of Study Participants according to Their Mycobacterium tuberculosis Infection Status. Sixty-four healthy household contacts of recently (within past 2 months) diagnosed active pulmonary TB patients underwent TST and 2 commercial IGRA tests, QFT, and T-SPOT.TB. Fifty-three household contacts were classified as LTBI due to positivity in TST (induration $\geq 15 \mathrm{~mm}$ ) [29], QFT and/or T-SPOT.TB ( $n=53$; age range: $15-59$ years; $58.2 \%$ females). The remaining 11 household contacts were classified as healthy controls (HC). As noninfected control individuals were rare in the described TB endemic settings [28], we added 3 healthy nonhousehold contacts (community controls with no known previous TB exposure) with TST-indurations of $0 \mathrm{~mm}$, normal chest X-rays and AFB-negative-assisted sputum samples to the healthy control group in order to increase the statistical power $(n=14$; age range: $10.7-$ 55.2 years; $71.4 \%$ females). The Mantoux induration $>15 \mathrm{~mm}$ criterion was used for the definition of LTBI, as a large-scale study performed in a rural African population showed that the local environmental mycobacterial exposure is reflected in Mantoux indurations that cluster around $10 \mathrm{~mm}$, whereas the M. tuberculosis exposures are reflected by indurations with a mode at $15-17 \mathrm{~mm}$ or larger [29]. Still, the exact definition of the LTBI status is hampered by the lack of a gold standard. According to Centers for Disease Control and Prevention (CDC) criteria Mantoux induration $>5 \mathrm{~mm}$ of recent TB household contacts can be considered as LTBI [30], and we alternatively considered this cut off for defining a positive tuberculin reaction: of the fourteen members of the original HC group, two subjects had Mantoux indurations between $5 \mathrm{~mm}$ and $15 \mathrm{~mm}$, and both individuals had negative IGRA results. Therefore, in an alternative grouping of the controls, we transferred the 2 IGRA-negative individuals with Mantoux indurations between 5 and $15 \mathrm{~mm}$ from the HC group (HC $\left.{ }^{*}: n=12\right)$ into the LTBI group (LTBI* $\left.: n=55\right)$. The 42 smear-positive active pulmonary TB patients (age range: $18-55$ years; $40.5 \%$ females) included in this study were recruited from the same community as the control participants.

3.2. Profile of Specific IgA and/or IgG Antibodies in an Endemic Setting. The 5 protein antigens NarL, AlaDH, $19 \mathrm{kDa}$, PstS3, and MPT83 (Table 1) were cloned and expressed in E. coli and purified using standard chromatographic methods. A 


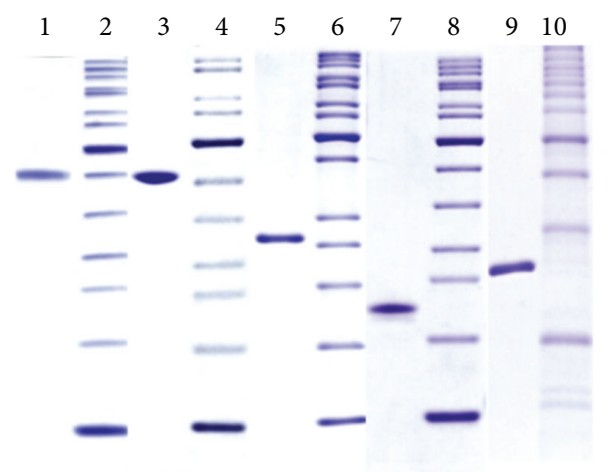

FIGURE 1: Quality control SDS-PAGE analysis (reduced) of the highly purified recombinant antigens ( $1 \mu \mathrm{g}$ per lane). Lane 1: PstS3, Lane 3: AlaDH, Lane 5: MPT83, Lane 7: $19 \mathrm{kDa}$, Lane 9: NarL, Lanes 2, 4, 6, 8, and 10: molecular weight ladders (each corresponding to the antigen on the left side), Lanes 1-4: 15\% Laemmli gels, and Lanes 5-10: 12\% Laemmli gels. All stained with Coomassie brilliant blue R250.

Coomassie Brilliant Blue R-250 stained SDS-PAGE analysis of the 5 proteins is shown in Figure 1.

The 109 serum samples derived from 42 pulmonary TB patients, 53 LTBI controls, and 14 non-LTBI controls were tested in a blinded fashion for IgA responses specific to the 5 proteins as well as for IgG responses to AlaDH. Among controls, we found that elevated IgA levels against the investigated 5 antigens were not randomly distributed but concentrated on a subgroup of $28.4 \%(n=19)$-with particular high levels in a subgroup of $4.5 \%(n=3)$, which comprised the progressor from latent infection to active TB. To graphically distinguish between those LTBI individuals with negative serum data (more than $70 \%$ of the controls) and those with moderately elevated and highly elevated serology, we used the mean ranks of IgA signals against the 5 antigens investigated to divide the LTBI subjects into the 3 subgroups LTBI (low IgA), LTBI (medium IgA) and LTBI (high IgA) (Figure 2). Furthermore, we statistically compared the non-LTBI controls with either the active TB patients or the LTBI subgroup with elevated IgA signals comprising the 2 subgroups LTBI (medium IgA) and LTBI (high IgA) (Table 2). Based on a cut-off referring to the 92.9percentile of the healthy non-LTBI controls $[92.9 \%$ (95\% CI, 66.1-99.8\%) specificity], the IgA response against the novel protein antigen NarL achieved with 81\% (95\% CI, 65.991.4\%) the highest sensitivity for the detection of active $\mathrm{TB}$ patients, followed by anti-AlaDH IgA with $76.2 \%$ (95\% CI, 60.5-87.9\%) sensitivity and anti-19 kDa IgA with 64.3\% (95\% CI, 48.0-78.4\%) sensitivity (Table 2). Moreover, based on a specificity of $100 \%$ (95\% CI, 76.8-100\%), anti-NarL IgA and anti-AlaDH IgA both detected 84.2\% (95\% CI, 60.4$96.6 \%)$ of the LTBI subgroup with elevated IgA signals, followed by anti-19 $\mathrm{kDa}$ IgA with 78.9\% (95\% CI, 54.4-93.9\%) sensitivity (Table 2). Anti-NarL IgA, anti-19 kDa IgA, and anti-AlaDH IgA detected the LTBI (high IgA) subgroup, which comprised the progressor to active $\mathrm{TB}$, with a distinct signal-to-noise ratio compared to the non-LTBI community controls (Figures 2(a), 2(b), and 2(f)). In contrast, the LTBI (high IgA) subgroup was indistinguishable from the nonLTBI community controls when using the IgG response to AlaDH (Figure 2(e)). Very similar results to those shown in Table 2 were obtained when using the alternative groups $\mathrm{HC}^{*}$ and LTBI* (Table S1).

When testing for correlations between the antibody OD values in LTBI sera $(n=53)$ (Table 3 and Figure 3$)$, we found strong correlations between the IgA responses and the 5 protein antigens [strongest correlation between antiAlaDH IgA and anti-19 kDa IgA: Spearman's $r=0.96(95 \%$ CI, 0.93-0.98); $P<0.0001$ (Figure 3(a)); weakest correlation between anti-NarL IgA and anti-PstS3 IgA: $r=0.82(95 \%$ CI, 0.71-0.89); $P<0.0001$ (Figure 3(d))]. In contrast, we found no or negligible correlations of any of the IgA responses with the exemplarily tested IgG response to $\mathrm{AlaDH}$ [e.g., no correlation between anti-AlaDH IgA and anti-AlaDH IgG: $r=0.21$ (95\% CI, $-0.06-0.46) ; P=0.128$ (Figure 3(c))] (Table 3). Very similar results to those shown in Table 3 were obtained when using the alternative group LTBI ${ }^{*}$ (Table S2).

\section{Discussion}

In recent years it became increasingly clear that antibodybased diagnostics of active TB (often developed and tested in non-TB endemic countries) performed poorly in TB endemic settings [10] due to high background signals of antibodies in LTBI individuals [11]. In our study we also found that the presence of LTBI affected identification of active TB by serology. In particular, we found that among controls elevated IgA levels against the investigated 5 antigens were not randomly distributed but concentrated on a subgroup of $<30 \%$-with particular high levels in a small subgroup of $\sim 5 \%$. The consistently elevated IgA levels in a subgroup of controls suggest higher mycobacterial load, a risk factor for progression to active TB. Whether high IgA and/or IgG levels have prognostic potential should be investigated in future large scale studies.

In several previous reports it has indeed been suggested, directly or indirectly, that progression to active TB may be predicted by increased specific antibodies levels. Certain TBspecific antibody responses decline significantly during successful therapy (and the time thereafter) when both bacterial load and risk of disease also decline [38, 39]. In inactive TB (defined by a positive response to the TST, negative sputum cultures, and abnormal but stable CXR findings), a special form of latent infection known to have increased risk of active $\mathrm{TB}$, elevated specific antibody responses have been reported $[40,41]$. Several independent studies showed that antibody responses to mycobacterial proteins were detectable months to years prior to the diagnosis of TB in persons infected with HIV, for example, [8], again suggesting that in vivo $M$. tuberculosis replication may begin long before progression to active TB becomes clinically detectable. Specific antibacterial antibodies have been shown to be present in sera obtained from M. tuberculosis $\mathrm{H} 37 \mathrm{Rv}$ aerosol-infected rabbits, and guinea pigs both with preclinical TB $[7,8]$. In mouse models of $M$. avium infection, susceptibility to infection correlated 


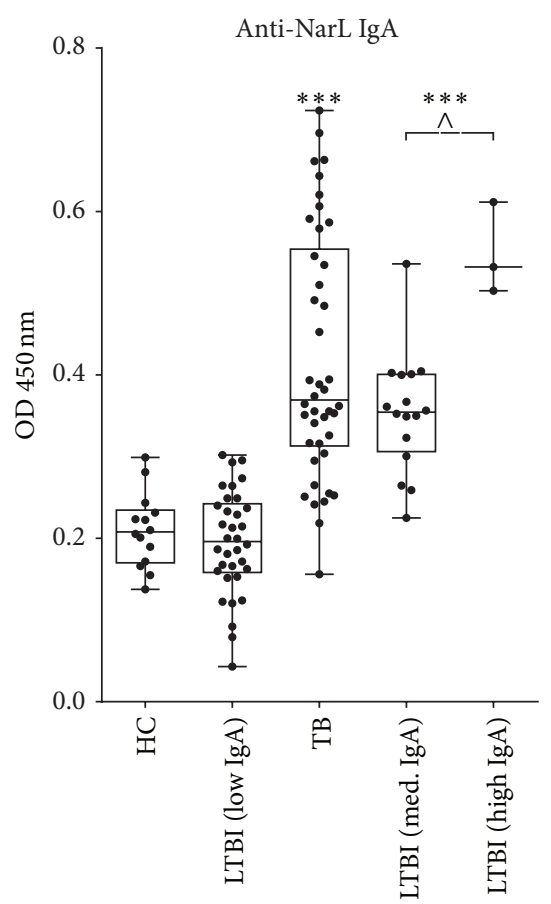

(a)

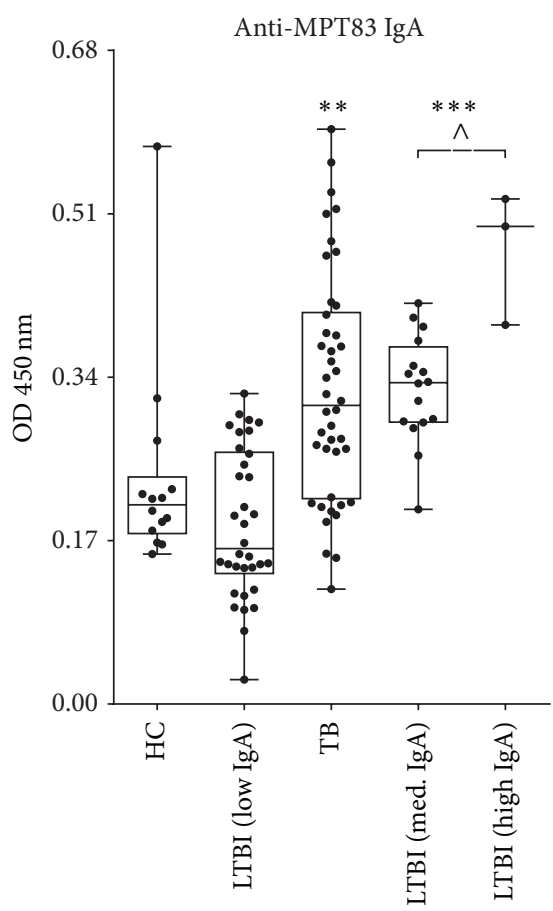

(d)

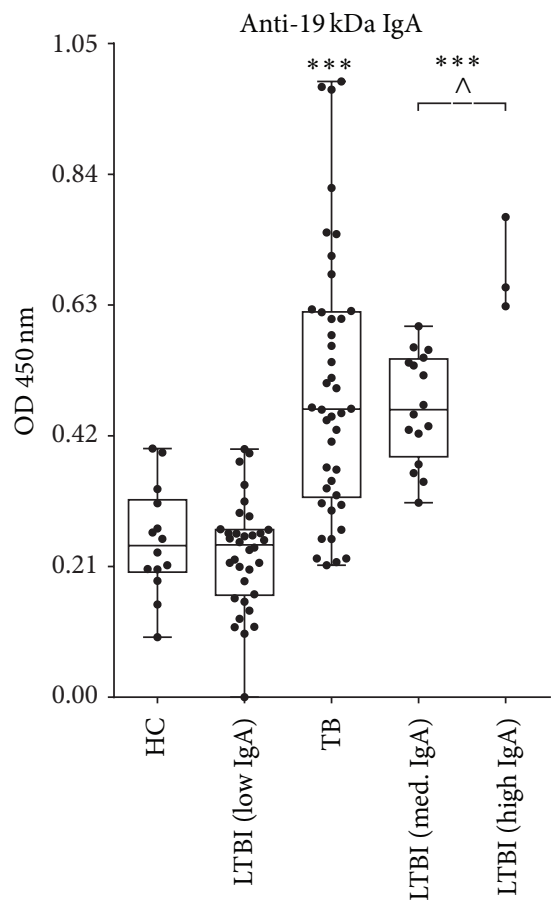

(b)

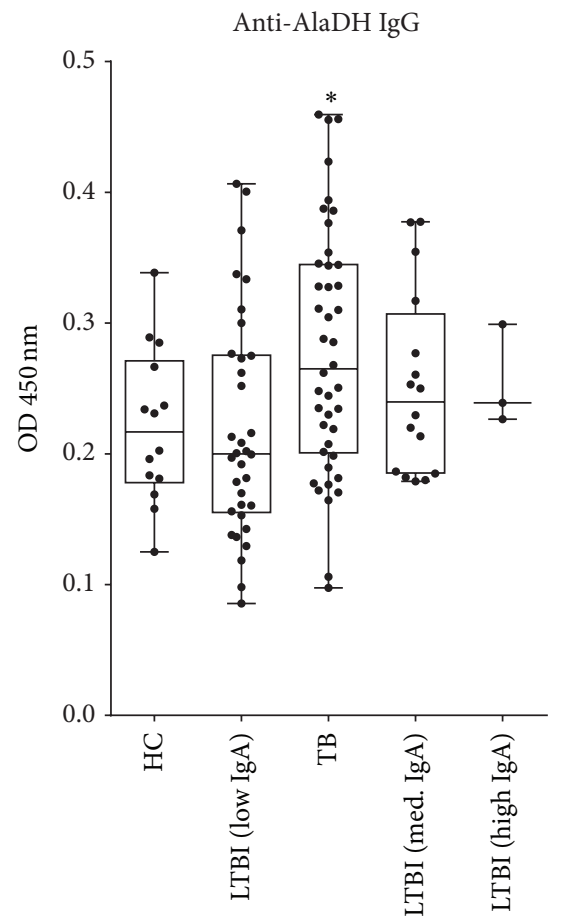

(e)

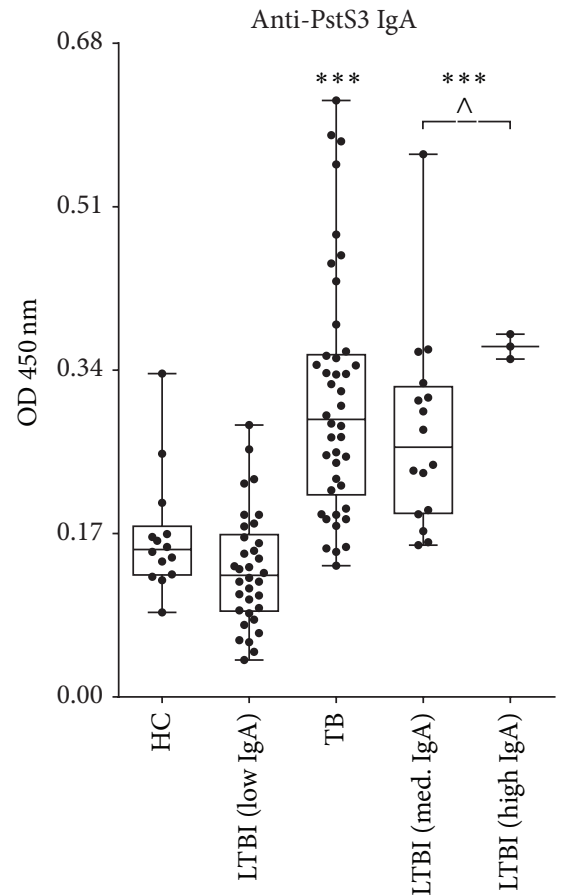

(c)

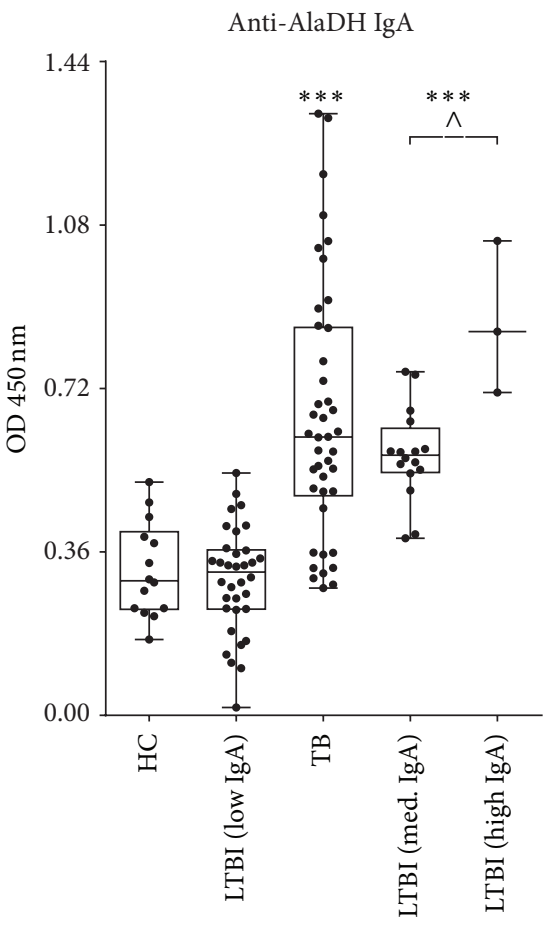

(f)

FIGURE 2: Box-and-whisker plots including individual points for the comparison of $109 \mathrm{~TB}$ or non-TB specimens showing the optical density (OD) values of the following selected antigens: anti-NarL IgA (a), anti-19 kDa IgA (b), anti-PstS3 IgA (c), anti-MPT83 IgA (d), anti-AlaDH $\operatorname{IgG}(\mathrm{e})$, and anti-AlaDH IgA (f). Values are shown for sera from 14 healthy controls (HC), 42 TB patients, and 3 different LTBI groups. The mean ranks of IgA signals against the 5 antigens investigated were used to group the LTBI group into the top 3 group [LTBI (high IgA); comprising the progressor to active TB disease], the following 16 mean ranks [LTBI (medium IgA)] and the remaining LTBI group [LTBI (low IgA)]. The symbol * depicted above either the TB patients or the LTBI (high and medium IgA) group shows a significant difference of this group compared to healthy controls. A $P$ value of $\leq 0.05$ was judged significant and levels of significance were indicated as follows: ${ }^{*} P=0.01-0.05,{ }^{* *} P=0.001-0.01,{ }^{* * *} P<0.001$. 
TABLE 2: Specificities and sensitivities of single seroantigens to distinguish between healthy non-TB infected controls [HC $(n=14)]$ and either active TB patients $(n=42)$ or LTBI [with medium or high IgA levels $(n=19)$ ].

\begin{tabular}{|c|c|c|c|c|c|}
\hline \multirow[b]{2}{*}{ Antigen } & \multirow[b]{2}{*}{ Ig class } & \multicolumn{2}{|c|}{ TB versus $\mathrm{HC}$} & \multicolumn{2}{|c|}{ LTBI (elevated IgA) versus HC } \\
\hline & & $\begin{array}{c}\text { Sens. }(\%)(95 \% \text { CI }) \text { based } \\
\text { on } 92.9 \%(95 \% \text { CI, } \\
66.1-99.8 \%) \text { spec. }\end{array}$ & $\begin{array}{c}\text { Sens. }(\%)(95 \% \text { CI }) \text { based } \\
\text { on } 100 \%(95 \% \text { CI, } \\
76.8-100 \%) \text { spec. }\end{array}$ & $\begin{array}{c}\text { Sens. }(\%)(95 \% \text { CI }) \text { based } \\
\text { on } 92.9 \%(95 \% \text { CI, } \\
66.1-99.8 \%) \text { spec. }\end{array}$ & $\begin{array}{c}\text { Sens. }(\%)(95 \% \text { CI }) \text { based } \\
\text { on } 100 \%(95 \% \text { CI, } \\
76.8-100 \%) \text { spec. }\end{array}$ \\
\hline NarL & $\mathrm{A}$ & $81.0(65.9-91.4)^{* * *}$ & $78.6(63.2-89.7)^{* * *}$ & $84.2(60.4-96.6)^{* * *}$ & $84.2(60.4-96.6)^{* * *}$ \\
\hline MPT83 & A & $47.6(32.0-63.6)^{\# \#}$ & $2.4(0.06-12.6)^{\# \#}$ & $63.2(38.4-83.7)^{\# \# \#}$ & $0(0.0-17.6)^{\# \# \#}$ \\
\hline $19 \mathrm{kDa}$ & A & $64.3(48.0-78.4)^{* * *}$ & $64.3(48.0-78.4)^{* * *}$ & $78.9(54.4-93.9)^{* * *}$ & $78.9(54.4-93.9)^{* * *}$ \\
\hline PstS3 & A & $61.9(45.6-76.4)^{* * *}$ & $35.7(21.6-52.0)^{* * *}$ & $57.9(33.5-79.7)^{* * *}$ & $31.6(12.6-56.6)^{* * *}$ \\
\hline $\mathrm{AlaDH}$ & G & $42.9(27.7-59.0)^{*}$ & $28.6(15.7-44.6)^{*}$ & $26.3(9.1-51.2)$ n. s. & $15.8(3.4-39.6)$ n.s. \\
\hline $\mathrm{AlaDH}$ & A & $76.2(60.5-87.9)^{* * *}$ & $69.0(52.9-82.4)^{* * *}$ & $89.5(66.9-98.7)^{* * *}$ & $84.2(60.4-96.6)^{* * *}$ \\
\hline
\end{tabular}

Significance levels of $P$ values: $P$ values refer to the $t$-test (if appropriate on log-transformed data) or the Mann-Whitney test where indicated. A $P$ value of $\leq 0.05$ was judged significant and levels of significance were indicated as follows: ${ }^{*}$ or ${ }^{\#} P=0.01-0.05,{ }^{* *}$ or ${ }^{\# \#} P=0.001-0.01,{ }^{* * *}$ or ${ }^{\# \# \#} P<0.001$; asterisks refer to parametric tests, hashes to nonparametric tests.

vs. = versus; n. s. = not significant; sens. = sensitivity; spec. $=$ specificity.

The mean ranks of the IgA signals against the 5 investigated antigens were used to group the LTBI group into the top 19 groups [LTBI with elevated mean IgA levels $(n=19)$ ] and the remaining LTBI group [LTBI (low IgA)].

The data were analyzed by using specificity levels of $\geq 90 \%$, as described by other investigators $[36,37]$.

TABLE 3: Spearman correlation between the antibody performances for 53 individuals with LTBI ${ }^{\mathrm{a}}$.

\begin{tabular}{|c|c|c|c|c|c|}
\hline Anti-AlaDH IgG & Anti-AlaDH IgA & Anti-19 kDa IgA & Anti-PstS3 IgA & Anti-MPT83 IgA & \\
\hline $\begin{array}{l}r=0.17(0.1-0.42) \\
P=0.22\end{array}$ & $\begin{array}{c}r=0.93(0.87-0.96) \\
P<0.0001\end{array}$ & $\begin{array}{c}r=0.91(0.85-0.95) \\
P<0.0001\end{array}$ & $\begin{array}{c}r=0.82(0.71-0.89) \\
P<0.0001\end{array}$ & $\begin{aligned} r= & 0.89(0.81-0.93) \\
& P<0.0001\end{aligned}$ & anti-NarL IgA \\
\hline - & $\begin{array}{c}r=0.21(-0.06-0.46) \\
P=0.13\end{array}$ & $\begin{array}{c}r=0.26(-0.01-0.49) \\
P=0.064\end{array}$ & $\begin{array}{c}r=0.31(0.04-0.53) \\
P=0.025\end{array}$ & $\begin{array}{c}r=0.27(0.0-0.50) \\
P=0.052\end{array}$ & anti-AlaDH IgG \\
\hline - & - & $\begin{array}{c}r=0.96(0.93-0.98) \\
P<0.0001\end{array}$ & $\begin{array}{c}r=0.86(0.77-0.92) \\
P<0.0001\end{array}$ & $\begin{array}{c}r=0.83(0.72-0.90) \\
P<0.0001\end{array}$ & anti-AlaDH IgA \\
\hline - & - & 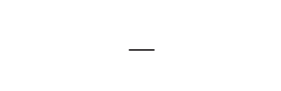 & $\begin{array}{c}r=0.88(0.80-0.93) \\
P<0.0001\end{array}$ & $\begin{array}{c}r=0.83(0.73-0.90) \\
P<0.0001\end{array}$ & anti-19 kDa IgA \\
\hline - & - & - & - & $\begin{array}{c}r=0.88(0.80-0.93) \\
P<0.0001\end{array}$ & anti-PstS3 IgA \\
\hline
\end{tabular}

${ }^{\mathrm{a}}$ Spearman's coefficient $r$ of rank correlation (95\% CI for $r$ ).

with increased synthesis of specific anti-bacterial antibodies [9]. Integration of macaque and human proteome-scale antibody profiling data revealed dynamic characteristics of the antibody response in relation to bacillary burden and infection outcome [4]. Two individuals with elevated specific IgG responses originally categorized as LTBI were subsequently diagnosed to have culture-confirmed active TB within a few weeks of the serologic testing [6]. Moreover, among apparently healthy professional contacts of TB patients (or pathological specimens thereof), elevated specific IgG and/or IgM responses to mycobacterial antigens were determined in a subgroup of 9 individuals, of whom 4 (44.4\%) developed active TB within one year after serological testing [5].

Clearly, future large scale, long term prospective studies in different TB endemic areas are needed to further evaluate and substantiate the validity of the hypothesis that certain specific anti-mycobacterial antibody responses are predictors of future active TB. If true, the reported low specificity of serodiagnostics for the detection of active TB would turn out to be due to a high risk for an early stage of progression to active disease and would then offer new opportunities to interrupt the cycle of transmission. If our findings are confirmed the clinical relevance would be as follows: a corresponding antibody-based test in endemic settings would then not differentiate between active TB cases on the one hand and latent or absent infection on the other hand. Instead, it would distinguish a high-risk group for preclinical or active TB from a group that is unlikely to progress to clinical disease. This would be of particular significance as such antibody tests could be developed into low-cost, point of care tests that may be used as screening tools, particularly in high TB burden low-resource settings. In such a clinical approach, the pre-screened high risk individuals could then be investigated using established assays such as X-ray, IGRA, sputum smear, sputum culture and GeneXpert MTB/RIF for final clinical assessment $[3,42]$. Concerning the serodiagnostic results, those LTBI individuals showing the highest IgA or IgG antibody signals might have the highest risk to progress to active $\mathrm{TB}$ as their higher antibody levels may indicate higher bacterial loads. The detection of preclinical and early TB would have considerable clinical impact, as the identification, close monitoring, or early treatment of those LTBI with incipient disease would offer an opportunity to break the cycle of transmission and to prevent more serious 


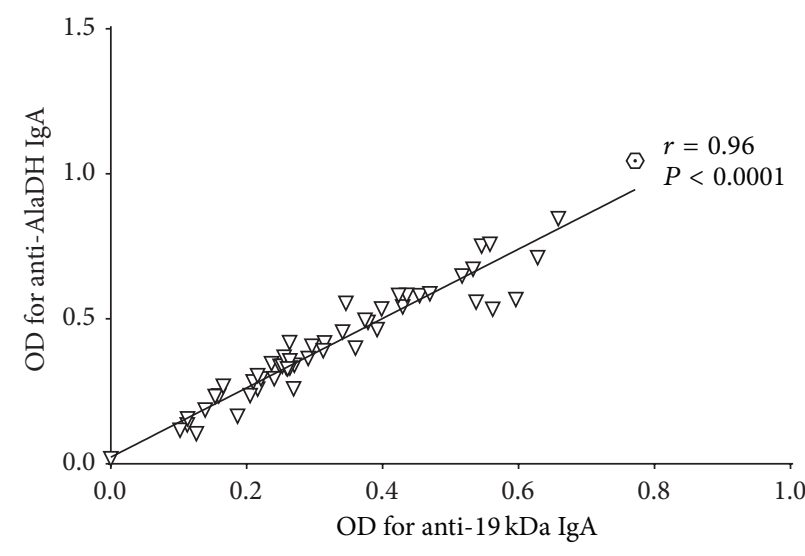

(a)

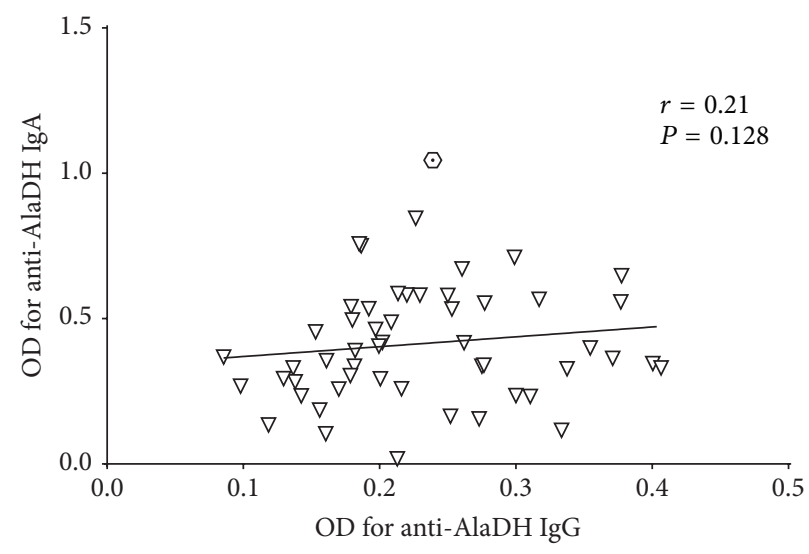

(c)

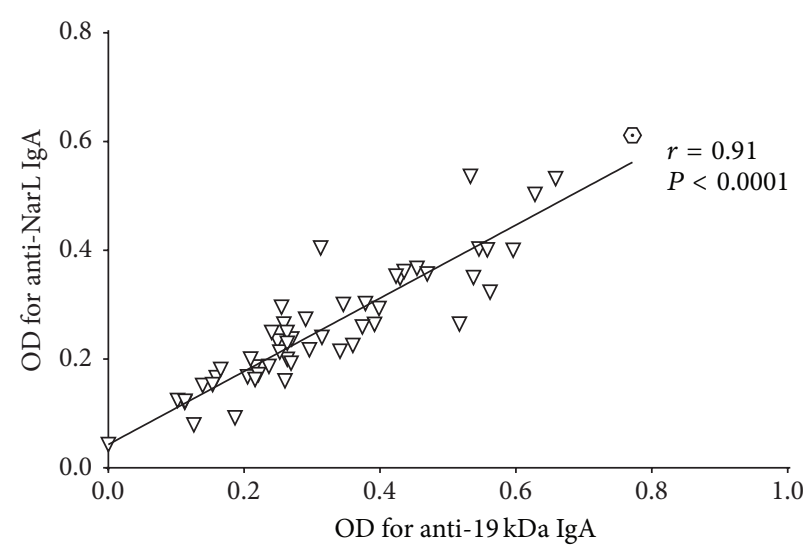

(b)

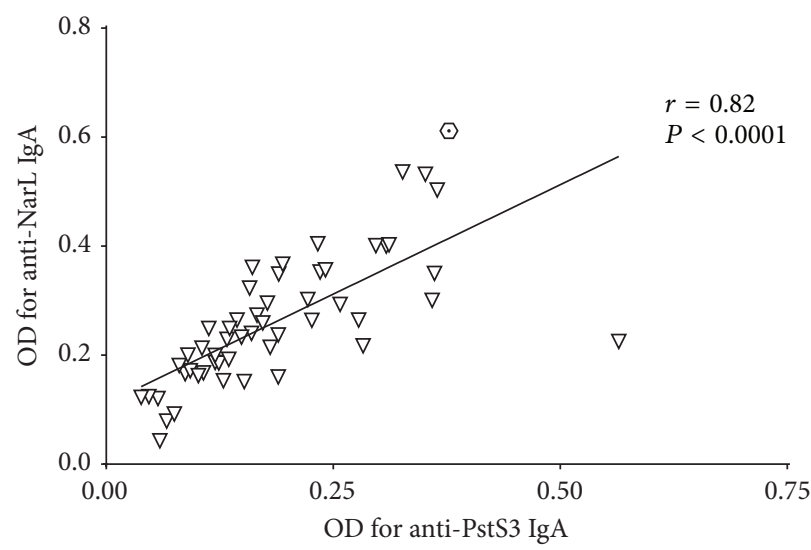

(d)

Figure 3: Correlations of IgA antibody levels in serum of latently M. tuberculosis infected individuals. Scatter graphs showing the relations between anti-AlaDH IgA and anti-19 kDa IgA (a), anti-NarL IgA and anti-19 kDa IgA (b) and anti-AlaDH IgA and anti-AlaDH IgG (c) as well as anti-NarL IgA and anti-PstS3 IgA (d) using the optical density (OD) values in diluted sera $(n=53)$. The correlation coefficient $r$ and the $P$ value were calculated using the Spearman rank test. The hexagon with a dot in its center characterizes the progressor to active TB, whereas all other latently $M$. tuberculosis infected individuals are characterized by the symbol $\nabla$.

lung destruction. In addition, early treatment of LTBI may decrease the emergence of multidrug resistance- (MDR-) TB strains [43], as the low numbers and slow turnover of mycobacteria in LTBI presumably present less opportunity to develop resistance.

In the present study the LTBI subject with the highest IgA levels against NarL, AlaDH and $19 \mathrm{kDa}$ developed active TB within 3 months after recruitment. Although this is in line with the working hypothesis, one has to be very careful with its interpretation as we are dealing only with a single case. Clearly, as stated above, further research is needed to investigate whether antibody responses to mycobacterial antigens hold any prognostic significance for subsequent development of active TB in individuals with LTBI as previously suggested $[4-6,8]$.

Though a recent metaanalysis showed that so far neither IGRAs nor the TST can discriminate the $~ 90 \%$ of persons with true LTBI from the $\sim 10 \%$ who will develop active TB [44], promising tendencies are noteworthy also in this field
$[45,46]$. Subjects with high risk for preclinical TB might be monitored more closely in defined time intervals using the currently best available resources and should be treated where applicable.

Due to the low frequency of reactivation in immunocompetent LTBI individuals even in TB endemic settings, large-scale longitudinal human studies would have to be conducted to further investigate whether serologic responses to mycobacterial antigens hold any prognostic significance for subsequent development of active TB in individuals with LTBI (correlates of risk). The results of this study and previous human and animal studies $[4,5,7-9,38-41]$ suggest that this is a promising approach, particularly as the serological tests could be performed on existing stored sera from previous studies. Our results suggest that for these investigations besides IgG also IgA serology should be considered. Due to the important role of IgA antibodies in the lung, the additional consideration of sputum IgA levels might be meaningful. The development of point-of-care tests that can 
be used as correlates of risk or as diagnostic for active TB would constitute a major advance.

\section{Conclusions}

In conclusion, as suggested in human studies and several animal models $[4,5,7-9,38-41]$, it remains a promising hypothesis that those latently infected individuals with antibody responses resembling those of active $\mathrm{TB}$ subjects are more prone to progression to active TB. Our finding of a nonrandom distribution of antibody responses among LTBI subjects suggests that the development of serodiagnostic kits for the determination of a high-risk group for preclinical or active TB in endemic settings may be possible. Furthermore, our results encourage the further investigation of IgA besides IgG responses in the field of serodiagnosis of active TB and possibly preclinical TB. Moreover, IgA antibodies against the novel antigen NarL were able to determine, besides the putative high-risk subgroup for preclinical TB (including highest levels for the actual progressor), the highest proportion of active TB patients.

\section{Abbreviations}

TB: Tuberculosis

LTBI: Latent Mycobacterium tuberculosis infection

HC: Healthy controls.

\section{Conflict of Interests}

The authors declare no conflicts of interests. LIONEX did not provide any financial support to the partners.

\section{Authors' Contribution}

Ralf Baumann and Susanne Kaempfer contributed equally to this work.

\section{Acknowledgments}

The authors would like to thank the Desmond Tutu TB Centre (Stellenbosch University), Wena Moelich, the data team, and the nursing sisters of the Desmond Tutu TB Centre for the recruitment of TB patients and controls with or without latent infection, the communities for their support and willingness to participate in the research, as well as the Department of Health and the City of Cape Town for their support. This work was partially supported by the Respiratory Research Unit, and by the DST/NRF Centre of Excellence for Biomedical and TB Research, University of Stellenbosch, Faculty of Medicine and Health Sciences, South Africa.

\section{References}

[1] C. Dye, S. Scheele, P. Dolin, V. Pathania, and M. C. Raviglione, "Consensus statement. Global burden of tuberculosis: estimated incidence, prevalence, and mortality by country. WHO Global Surveillance and Monitoring Project," Journal of the American Medical Association, vol. 282, no. 7, pp. 677-686, 1999.
[2] Stop TB partnership, The global plan to stop TB (2011-2015), 2011.

[3] B. D. Robertson, D. Altmann, C. Barry et al., "Detection and treatment of subclinical tuberculosis," Tuberculosis, vol. 92, no. 6, pp. 447-452, 2012.

[4] S. Kunnath-Velayudhan, A. L. Davidow, H.-Y. Wang et al., "Proteome-scale antibody responses and outcome of Mycobacterium tuberculosis infection in nonhuman primates and in tuberculosis patients," The Journal of Infectious Diseases, vol. 206, no. 5, pp. 697-705, 2012.

[5] H. L. David, F. Papa, P. Cruaud et al., "Relationships between titers of antibodies immunoreacting against glycolipid antigens from Mycobacterium leprae and M. tuberculosis, the Mitsuda and Mantoux reactions, and bacteriological loads: implications in the pathogenesis, epidemiology and serodiagnosis of leprosy and tuberculosis," International Journal of Leprosy, vol. 60, no. 2, pp. 208-224, 1992.

[6] E. D. Chan, R. Reves, J. T. Belisle, P. J. Brennan, and W. E. Hahn, "Diagnosis of tuberculosis by a visually detectable immunoassay for lipoarabinomannan," American Journal of Respiratory and Critical Care Medicine, vol. 161, no. 5, pp. 17131719, 2000.

[7] K. K. Singh, X. Zhang, A. S. Patibandla, P. Chien, and S. Laal, "Antigens of Mycobacterium tuberculosis expressed during preclinical tuberculosis: serological immunodominance of proteins with repetitive amino acid sequences," Infection and Immunity, vol. 69, no. 6, pp. 4185-4191, 2001.

[8] K. K. Singh, Y. Dong, S. A. Patibandla, D. N. McMurray, V. K. Arora, and S. Laal, "Immunogenicity of the Mycobacterium tuberculosis PPE55 (Rv3347c) protein during incipient and clinical tuberculosis," Infection and Immunity, vol. 73, no. 8, pp. 5004-5014, 2005.

[9] P. Ferreira, R. Soares, and M. Arala-Chaves, "Susceptibility to infection with Mycobacterium avium is paradoxically correlated with increased synthesis of specific anti-bacterial antibodies," International Immunology, vol. 3, no. 5, pp. 445452, 1991.

[10] K. R. Steingart, L. L. Flores, N. Dendukuri et al., "Commercial serological tests for the diagnosis of active pulmonary and extrapulmonary tuberculosis: an updated systematic review and meta-analysis," PLoS Medicine, vol. 8, no. 8, Article ID e1001062, 2011.

[11] S. T. Hoff, M. Abebe, P. Ravn et al., "Evaluation of Mycobacterium tuberculosis-specific antibody responses in populations with different levels of exposure from Tanzania, Ethiopia, Brazil, and Denmark," Clinical Infectious Diseases, vol. 45, no. 5, pp. 575-582, 2007.

[12] H. Diaz-Silvestre, P. Espinosa-Cueto, A. Sanchez-Gonzalez et al., "The 19-kDa antigen of Mycobacterium tuberculosis is a major adhesin that binds the mannose receptor of THP-1 monocytic cells and promotes phagocytosis of mycobacteria," Microbial Pathogenesis, vol. 39, no. 3, pp. 97-107, 2005.

[13] J. Arko-Mensah, E. Julián, M. Singh, and C. Fernández, “TLR2 but not TLR4 signalling is critically involved in the inhibition of IFN- $\gamma$-induced killing of mycobacteria by murine macrophages," Scandinavian Journal of Immunology, vol. 65, no. 2, pp. 148-157, 2007.

[14] A. Sánchez, P. Espinosa, T. García, and R. Mancilla, "The 19 $\mathrm{kDa}$ Mycobacterium tuberculosis lipoprotein (LpqH) induces macrophage apoptosis through extrinsic and intrinsic pathways: a role for the mitochondrial apoptosis-inducing factor," 
Clinical and Developmental Immunology, vol. 2012, Article ID 950503, 11 pages, 2012.

[15] F. F. Kao, S. Mahmuda, R. Pinto, J. A. Triccas, N. P. West, and W. J. Britton, "The secreted lipoprotein, MPT83, of Mycobacterium tuberculosis is recognized during human tuberculosis and stimulates protective immunity in mice," PLoS ONE, vol. 7, no. 5, Article ID e34991, 2012.

[16] R. Baumann, S. Kaempfer, N. N. Chegou et al., "Serodiagnostic markers for the prediction of the outcome of intensive phase tuberculosis therapy," Tuberculosis, vol. 93, no. 2, pp. 239-245, 2013.

[17] C. Palma, R. Spallek, G. Piccaro et al., "The M. tuberculosis phosphate-binding lipoproteins PstS1 and PstS3 induce Th1 and Th17 responses that are not associated with protection against M. tuberculosis infection," Clinical and Developmental Immunology, vol. 2011, Article ID 690328, 11 pages, 2011.

[18] F. Abebe, C. Holm-Hansen, H. G. Wiker, and G. Bjune, "Progress in serodiagnosis of Mycobacterium tuberculosis infection," Scandinavian Journal of Immunology, vol. 66, no. 2-3, pp. 176-191, 2007.

[19] S. Kunnath-Velayudhan, H. Salamon, H.-Y. Wang et al., "Dynamic antibody responses to the Mycobacterium tuberculosis proteome," Proceedings of the National Academy of Sciences of the United States of America, vol. 107, no. 33, pp. 14703-14708, 2010.

[20] R. Schnell, D. Ågren, and G. Schneider, "1.9 A structure of the signal receiver domain of the putative response regulator NarL from Mycobacterium tuberculosis," Acta Crystallographica F: Structural Biology and Crystallization Communications, vol. 64, pp. 1096-1100, 2008.

[21] G. A. de Souza, N. A. Leversen, H. Målen, and H. G. Wiker, "Bacterial proteins with cleaved or uncleaved signal peptides of the general secretory pathway," Journal of Proteomics, vol. 75, no. 2, pp. 502-510, 2011.

[22] A. Azzurri, G. V. Kanaujia, O. Y. Sow et al., "Serological markers of pulmonary tuberculosis and of response to anti-tuberculosis treatment in a patient population in Guinea," International Journal of Immunopathology and Pharmacology, vol. 19, no. 1, pp. 199-208, 2006.

[23] J. Mattow, P. R. Jungblut, U. E. Schaible et al., "Identification of proteins from Mycobacterium tuberculosis missing in attenuated Mycobacterium bovis BCG strains," Electrophoresis, vol. 22, pp. 2936-2946, 2001.

[24] M. Legesse, G. Ameni, G. Medhin et al., "IgA response to ESAT-6/CFP-10 and Rv2031 antigens varies in patients with culture-confirmed pulmonary tuberculosis, healthy mycobacterium tuberculosis-infected and non-infected individuals in a tuberculosis endemic setting, Ethiopia," Scandinavian Journal of Immunology, vol. 78, no. 3, pp. 266-274, 2013.

[25] M. B. Conde, P. Suffys, J. R. Lapa E Silva, A. L. Kritski, and S. E. Dorman, "Immunoglobulin A (IgA) and IgG immune responses against P-90 antigen for diagnosis of pulmonary tuberculosis and screening for Mycobacterium tuberculosis infection," Clinical and Diagnostic Laboratory Immunology, vol. 11, no. 1, pp. 94-97, 2004.

[26] C. O. Elson, J. A. Heck, and W. Stober, "T-cell regulation of murine IgA synthesis," Journal of Experimental Medicine, vol. 149, no. 3, pp. 632-643, 1979.

[27] N. Alvarez, O. Otero, F. Camacho et al., "Passive administration of purified secretory IgA from human colostrum induces protection against Mycobacterium tuberculosis in a murine model of progressive pulmonary infection," BMC immunology, vol. 14, supplement 1, article S3, 2013.

[28] S. den Boon, S. W. P. van Lill, M. W. Borgdorff et al., "Association between smoking and tuberculosis infection: a population survey in a high tuberculosis incidence area," Thorax, vol. 60, no. 7, pp. 555-557, 2005.

[29] P. E. M. Fine, J. Bruce, J. M. Ponnighaus, P. Nkhosa, A. Harawa, and E. Vynnycky, "Tuberculin sensitivity: conversions and reversions in a rural African population," International Journal of Tuberculosis and Lung Disease, vol. 3, no. 11, pp. 962975, 1999.

[30] "Targeted tuberculin testing and treatment of latent tuberculosis infection. American Thoracic Society," MMWRRecommendations and Reports, vol. 49, pp. 1-51, 2000.

[31] G. Ferrara, M. Losi, R. D’Amico et al., "Use in routine clinical practice of two commercial blood tests for diagnosis of infection with Mycobacterium tuberculosis: a prospective study," The Lancet, vol. 367, no. 9519, pp. 1328-1334, 2006.

[32] D. Vassilopoulos, S. Tsikrika, C. Hatzara et al., "Comparison of two gamma interferon release assays and tuberculin skin testing for tuberculosis screening in a cohort of patients with rheumatic diseases starting anti-tumor necrosis factor therapy," Clinical and Vaccine Immunology, vol. 18, no. 12, pp. 2102-2108, 2011.

[33] T. Roberts, N. Beyers, A. Aguirre, and G. Walzl, "Immunosuppression during active tuberculosis is characterized by decreased interferon- $\gamma$ production and CD25 expression with elevated forkhead box P3, transforming growth factor- $\beta$, and interleukin-4 mRNA levels," Journal of Infectious Diseases, vol. 195, no. 6, pp. 870-878, 2007.

[34] G. F. Black, B. A. Thiel, M. O. Ota et al., "Immunogenicity of novel DosR regulon-encoded candidate antigens of mycobacterium tuberculosis in three high-burden populations in Africa," Clinical and Vaccine Immunology, vol. 16, no. 8, pp. 1203-1212, 2009.

[35] B. Hutter and M. Singh, "Properties of the $40 \mathrm{kDa}$ antigen of Mycobacterium tuberculosis, a functional L-alanine dehydrogenase," Biochemical Journal, vol. 343, part 3, pp. 669-672, 1999.

[36] S. Laal, K. M. Samanich, M. G. Sonnenberg, S. Zolla-Pazner, J. M. Phadtare, and J. T. Belisle, "Human humoral responses to antigens of Mycobacterium tuberculosis: immunodominance of high-molecular-mass antigens," Clinical and Diagnostic Laboratory Immunology, vol. 4, no. 1, pp. 49-56, 1997.

[37] I. Rosenkrands, C. Aagaard, K. Weldingh et al., "Identification of Rv0222 from RD4 as a novel serodiagnostic target for tuberculosis," Tuberculosis, vol. 88, no. 4, pp. 335-343, 2008.

[38] S. Kitada, R. Maekura, N. Toyoshima et al., "Use of glycopeptidolipid core antigen for serodiagnosis of Mycobacterium avium complex pulmonary disease in immunocompetent patients," Clinical and Diagnostic Laboratory Immunology, vol. 12, no. 1, pp. 44-51, 2005.

[39] M. S. Imaz and E. Zerbini, "Antibody response to culture filtrate antigens of Mycobacterium tuberculosis during and after treatment of tuberculosis patients," The International Journal of Tuberculosis and Lung Disease, vol. 4, no. 6, pp. 562-569, 2000.

[40] V. M. C. Silva, G. Kanaujia, M. L. Gennaro, and D. Menzies, "Factors associated with humoral response to ESAT-6, $38 \mathrm{kDa}$ and $14 \mathrm{kDa}$ in patients with a spectrum of tuberculosis," International Journal of Tuberculosis and Lung Disease, vol. 7, no. 5, pp. 478-484, 2003.

[41] A. Davidow, G. V. Kanaujia, L. Shi et al., "Antibody profiles characteristic of Mycobacterium tuberculosis infection state," Infection and Immunity, vol. 73, no. 10, pp. 6846-6851, 2005. 
[42] J. M. Achkar, Y. Dong, R. S. Holzman et al., "Mycobacterium tuberculosis malate synthase- and MPT51-based serodiagnostic assay as an adjunct to rapid identification of pulmonary tuberculosis," Clinical and Vaccine Immunology, vol. 13, no. 11, pp. 1291-1293, 2006.

[43] M. Klopper, R. M. Warren, C. Hayes et al., "Emergence and spread of extensively and totally drug-resistant tuberculosis, South Africa," Emerging Infectious Diseases, vol. 19, no. 3, pp. 449-455, 2013.

[44] M. X. Rangaka, K. A. Wilkinson, J. R. Glynn et al., "Predictive value of interferon- $\gamma$ release assays for incident active tuberculosis: a systematic review and meta-analysis," The Lancet Infectious Diseases, vol. 12, no. 1, pp. 45-55, 2012.

[45] T. M. Doherty, A. Demissie, J. Olobo et al., "Immune responses to the Mycobacterium tuberculosis-specific antigen ESAT-6 signal subclinical infection among contacts of tuberculosis patients," Journal of Clinical Microbiology, vol. 40, no. 2, pp. 704706, 2002.

[46] S. Machingaidze, S. Verver, H. Mulenga et al., "Predictive value of recent quantiFERON conversion for tuberculosis disease in adolescents," The American Journal of Respiratory and Critical Care Medicine, vol. 186, no. 10, pp. 1051-1056, 2012. 


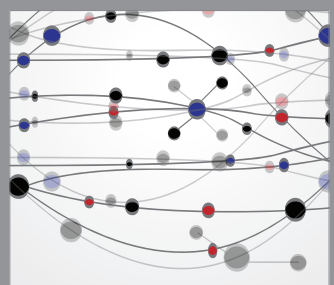

The Scientific World Journal
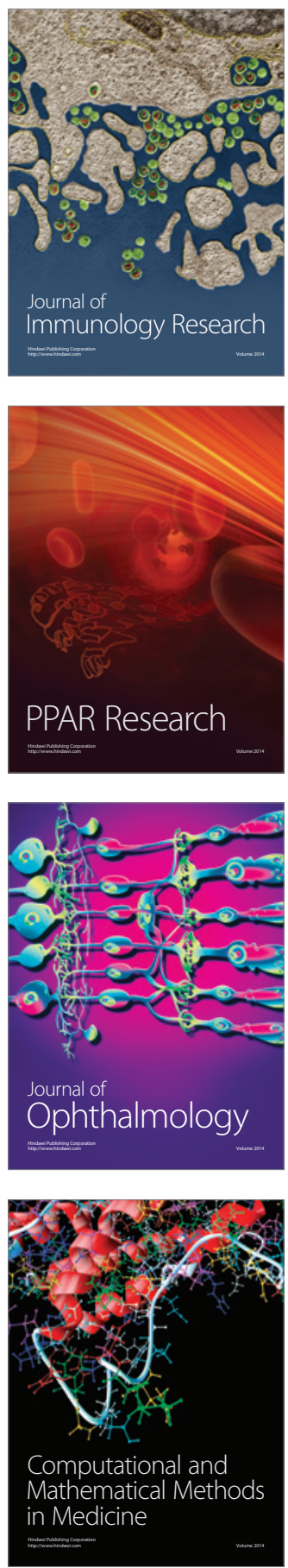

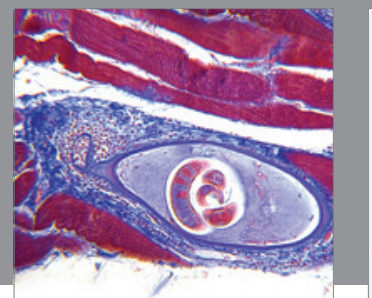

Gastroenterology

Research and Practice
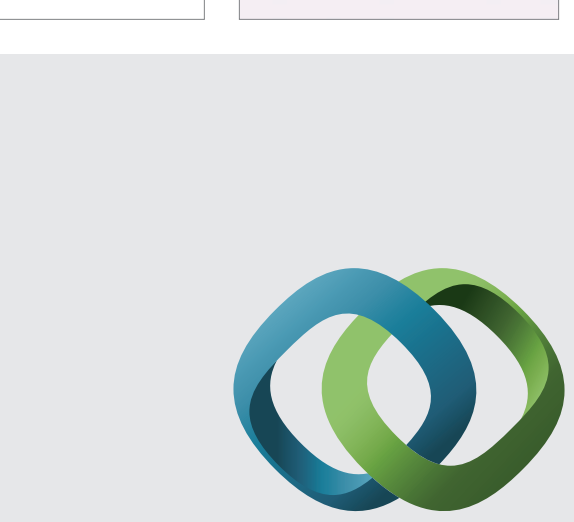

\section{Hindawi}

Submit your manuscripts at

http://www.hindawi.com
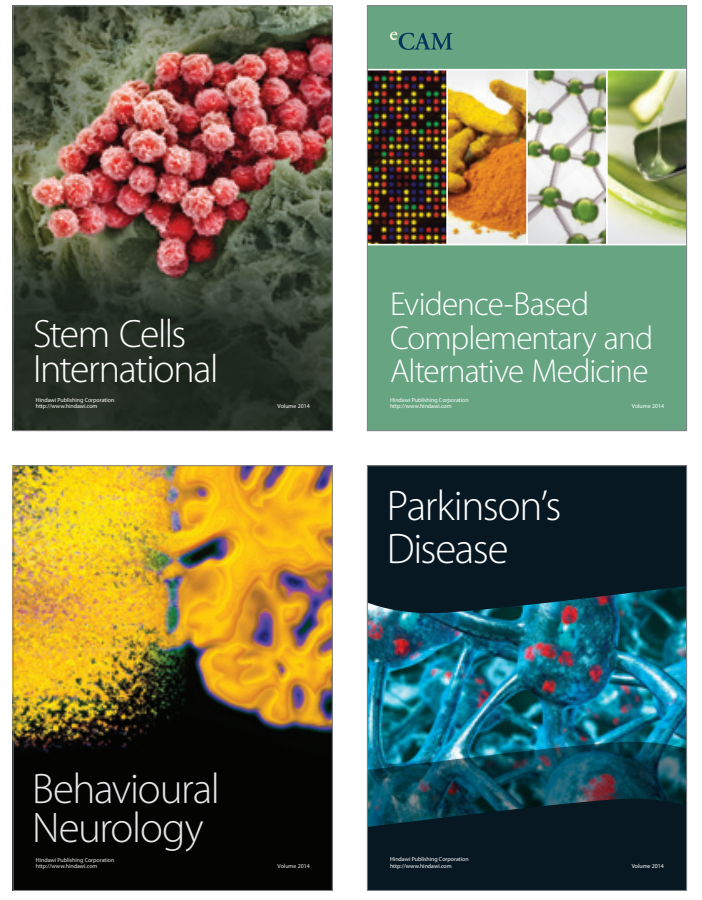
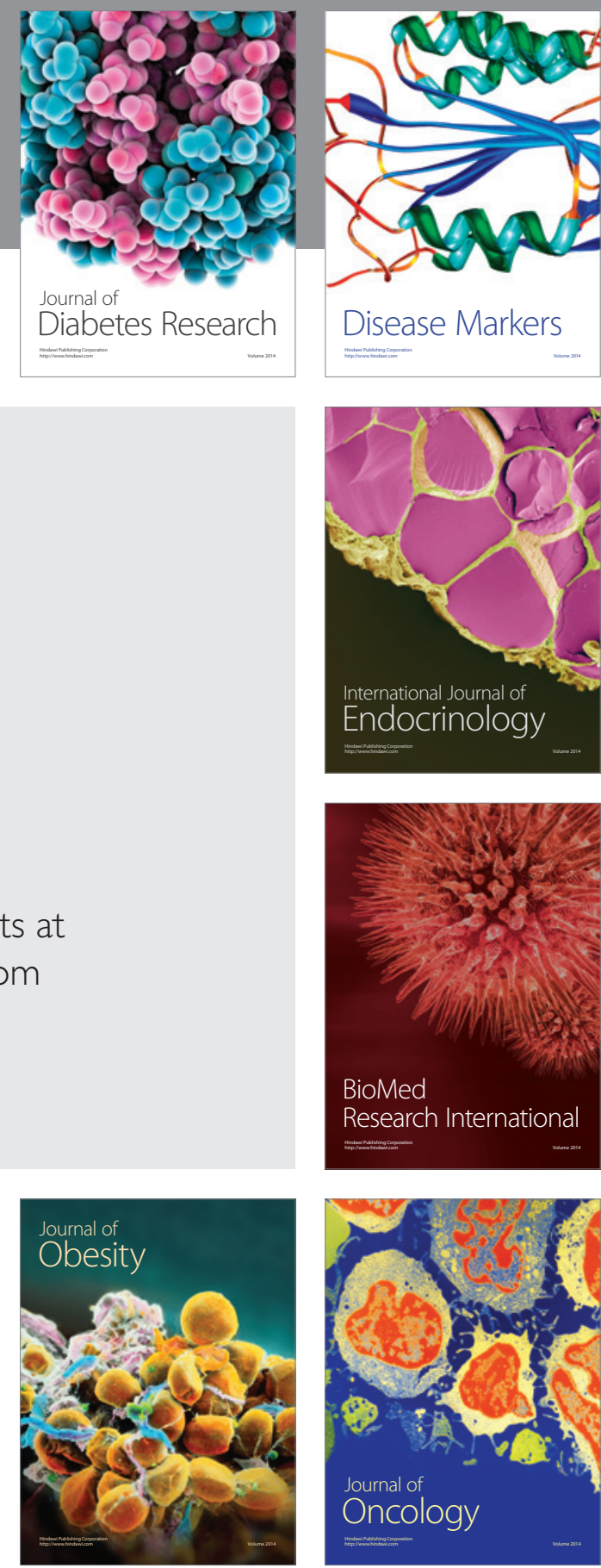

Disease Markers
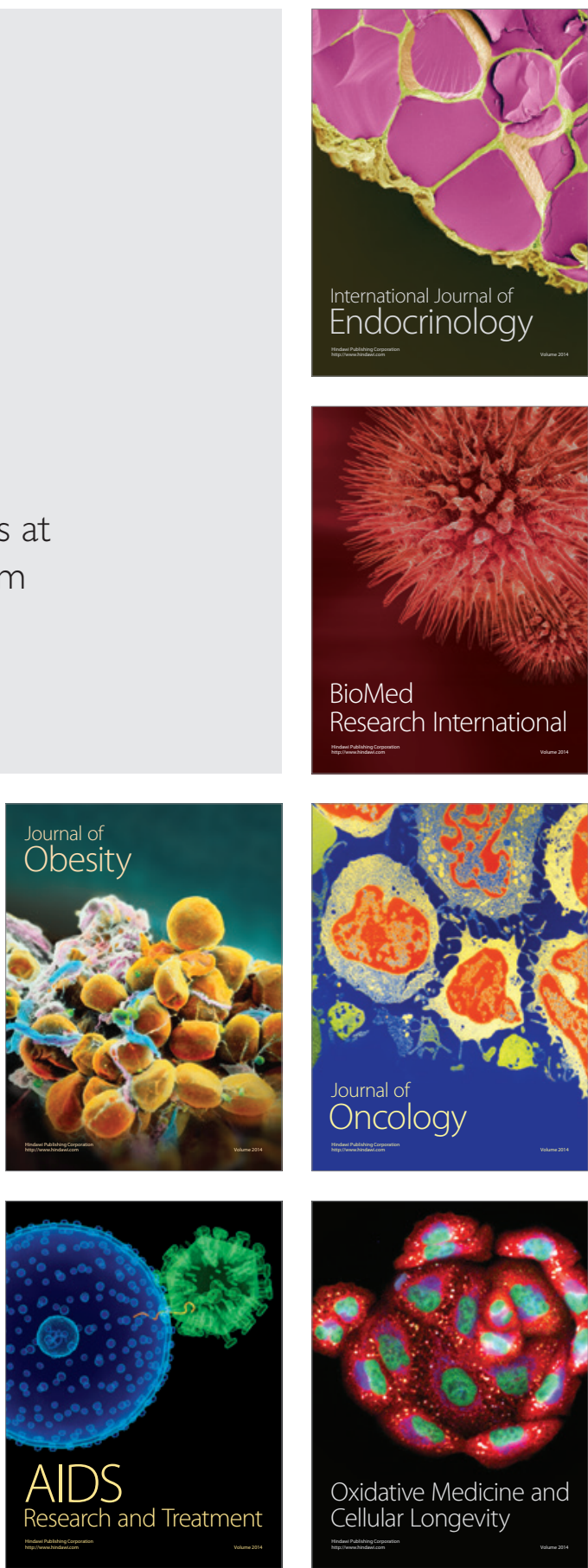